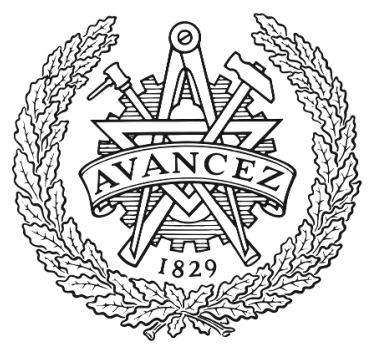

CHALMERS

UNIVERSITY OF TECHNOLOGY

\title{
Cation Distributions and Magnetic Properties of Ferrispinel MgFeMnO4
}

Downloaded from: https://research.chalmers.se, 2023-04-26 10:48 UTC

Citation for the original published paper (version of record):

Matsubara, N., Masese, T., Suard, E. et al (2020). Cation Distributions and Magnetic Properties of Ferrispinel MgFeMnO4. Inorganic Chemistry, 59(24): 17970-17980.

http://dx.doi.org/10.1021/acs.inorgchem.0c02241

N.B. When citing this work, cite the original published paper. 


\title{
Cation Distributions and Magnetic Properties of Ferrispinel $\mathrm{MgFeMnO}_{4}$
}

\author{
Nami Matsubara,* Titus Masese, Emmanuelle Suard, Ola Kenji Forslund, Elisabetta Nocerino, \\ Rasmus Palm, Zurab Guguchia, Daniel Andreica, Alexandra Hardut, Motoyuki Ishikado, \\ Konstantinos Papadopoulos, Yasmine Sassa, and Martin Månsson*
}

Cite This: Inorg. Chem. 2020, 59, 17970-17980

Read Online

\section{ACCESS | \\ WIII Metrics \& More \\ Article Recommendations \\ Supporting Information}

ABSTRACT: The crystal structure and magnetic properties of the cubic spinel $\mathrm{MgFeMnO}_{4}$ were studied by using a series of in-house techniques along with large-scale neutron diffraction and muon spin rotation spectroscopy in the temperature range between 1.5 and $500 \mathrm{~K}$. The detailed crystal structure is successfully refined by using a cubic spinel structure described by the space group $F d \overline{3} m$. Cations within tetrahedral $A$ and octahedral $B$ sites of the spinel were found to be in a disordered state. The extracted fractional site occupancies confirm the presence of antisite defects, which are of

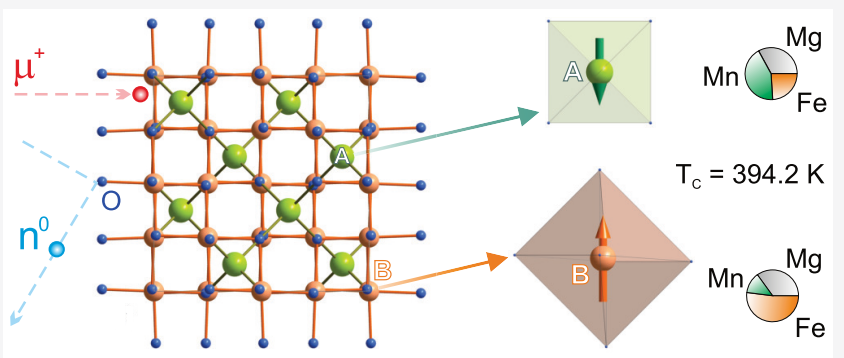
importance for the electrochemical performance of $\mathrm{MgFeMnO}_{4}$ and related battery materials. Neutron diffraction and muon spin spectroscopy reveal a ferrimagnetic order below $T_{\mathrm{C}}=394.2 \mathrm{~K}$, having a collinear spin arrangement with antiparallel spins at the $A$ and $B$ sites, respectively. Our findings provide new and improved understanding of the fundamental properties of the ferrispinel materials and of their potential applications within future spintronics and battery devices.

\section{INTRODUCTION}

The well-established spinel magnesium oxide family $\left(\mathrm{MgM}_{2} \mathrm{O}_{4}\right.$ where $M$ is a transition metal atom) has been the subject of interest within a broad scope of research as well as applications, for example, catalysts, humidity sensors, or microwave devices. ${ }^{1-4}$ Recently, increased attention has been drawn to a series of $\mathrm{Mg}$ materials, following the development of highenergy-density rechargeable magnesium batteries. ${ }^{5-7}$ However, spinel magnesium oxides also have many interesting fundamental materials properties, including diverse magnetic spin orders. This class of materials displays paramagnetism, ferrimagnetism, superparamagnetism, and spin-glass behavior. Such magnetic properties also play an essential role in the application within, for example, magnetic resonance imaging, data storage, and drug delivery systems. ${ }^{4,8}$

Spinels have the general formula $A B_{2} \mathrm{O}_{4}$, and most of them are reported to have a cubic crystal structure with space group $F d \overline{3} m$. The structure consists of tetrahedrally coordinated $A$ site cations at Wyckoff position $8 a(1 / 8,1 / 8,1 / 8)$ and octahedrally coordinated $B$ site cations at $16 d(1 / 2,1 / 2,1 / 2)$. Oxygen $(\mathrm{O})$ atoms lie on the body diagonals of the cube at $32 e$ $(u, u, u)$, where $u$ is approximately $1 / 4$. In the case of magnesium oxides, divalent magnesium and trivalent transition metal atoms tend to occupy the $A$ and $B$ sites, respectively. However, the cation distribution between the $A$ and $B$ sites, which is known to strongly influence the physical properties of these materials, ${ }^{9,10}$ is a controversial issue.
The parent compounds, $\mathrm{MgMn}_{2} \mathrm{O}_{4}$ and $\mathrm{MgFe}_{2} \mathrm{O}_{4}$, crystallize in different space groups and display very different magnetic properties. This was the main motivation for studying the solid solution $\left(\mathrm{MgFeMnO}_{4}\right)$ of these two compounds. $\mathrm{MgFe}_{2} \mathrm{O}_{4}$ crystallizes in the typical cubic spinel structure, with a slight $\mathrm{Mg} / \mathrm{Fe}$ disorder between the $A$ and $B$ sites. ${ }^{11,12}$ The magnetic ground state of $\mathrm{MgFe}_{2} \mathrm{O}_{4}$ has been reported to be a ferrimagnet below $T_{\mathrm{C}}=363-493 \mathrm{~K}$ (here $T_{\mathrm{C}}$ is strongly dependent on the cation distribution.). ${ }^{13,14}$ On the other hand, $\mathrm{MgMn}_{2} \mathrm{O}_{4}$ is known to form a distorted tetragonal phase (space group: $I 4_{1} /$ amd) due to the Jahn-Teller effect of $\mathrm{Mn}^{3+}{ }^{15-17}$ Typically, this compound shows a perfect cation ordering with the lattice sites being occupied as $A=\mathrm{Mg}^{2+}$ and $B=\mathrm{Mn}^{3+}$. In addition, an antiferromagnetic spin order occurs below $T_{\mathrm{N}}=50 \mathrm{~K}^{3}$. Recently, $\mathrm{Mg}\left(\mathrm{Mn}_{1-x} \mathrm{Fe}_{x}\right)_{2} \mathrm{O}_{4}$ has been synthesized and characterized by electrochemical measurements. Han et al. $^{6}$ reported that more than $20 \%$ of $\mathrm{Fe}$ substitution could stabilize the cubic spinel structure of $\mathrm{Mg}\left(\mathrm{Mn}_{1-x} \mathrm{Fe}_{x}\right)_{2} \mathrm{O}_{4}$, demonstrating that the charge compensation during the charge/discharge processes mainly occurs on

Received: July 28, 2020

Published: December 2, 2020 

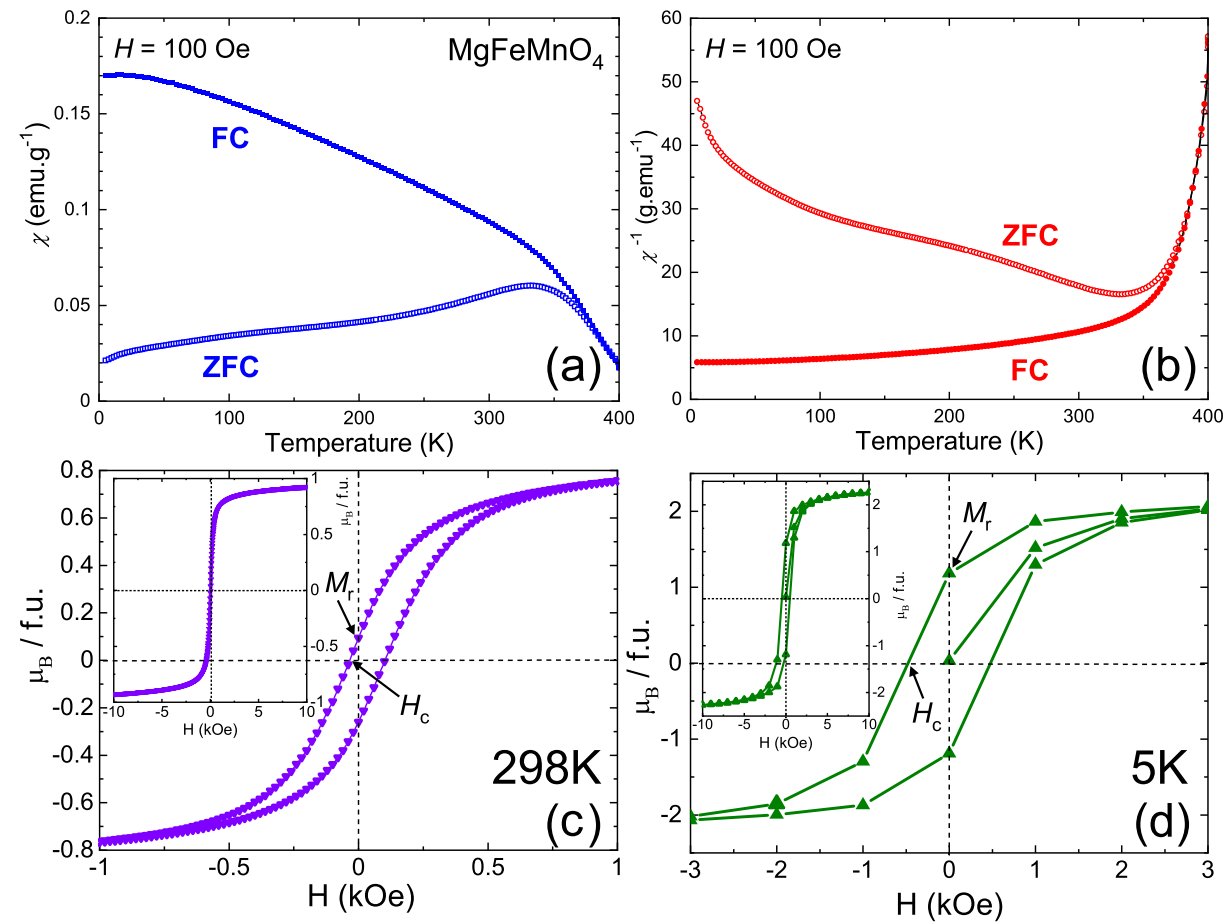

Figure 1. (a) Temperature dependence of the magnetic susceptibility for $\mathrm{MgFeMnO}_{4}$ recorded in $\mathrm{ZFC}$ and $\mathrm{FC}$ protocols under an external magnetic field $H=100$ Oe. (b) The corresponding inverse susceptibility $1 / \chi(T)$. (c, d) Magnetization vs magnetic field curves recorded at $(c) T=$ $298 \mathrm{~K}$ and (d) $T=5 \mathrm{~K}$. For (c, d) the inset shows the entire field range while main panels display a zoomed-in view of the most relevant field range where the coercivity $\left(H_{\mathrm{C}}\right)$ and retentivity $\left(M_{\mathrm{r}}\right)$ are highlighted.

$\mathrm{Mn}^{2+} / \mathrm{Mn}^{3+}$. However, the electrochemical performance of $\mathrm{MgMn}_{2} \mathrm{O}_{4}$ is not efficient because of the structural transition during the charge/discharge process between the tetragonal and the cubic phases. Thus, the substituted cubic system $\mathrm{Mg}\left(\mathrm{Mn}_{1-x} \mathrm{Fe}_{x}\right)_{2} \mathrm{O}_{4}$ is better suited as a magnesium battery cathode material. The detailed crystal structure and the magnetic characterization of $\mathrm{Mg}\left(\mathrm{Mn}_{1-x} \mathrm{Fe}_{x}\right)_{2} \mathrm{O}_{4}$ are missing so far to the best of our knowledge.

In this article, we present the detailed crystal structure and magnetic properties of the cubic spinel $\mathrm{MgFeMnO}_{4}$ (i.e., $x=$ $0.5)$. The cationic arrangement of the compound was studied by the combination of X-rays and neutron diffraction methods. Contrary to the X-ray diffraction, the differences in the neutron scattering amplitudes (i.e., contrast) of the constituent atoms have allowed an accurate determination of the cation distribution in $\mathrm{MgFeMnO}_{4}$. These crystallographic studies unveiled that $\mathrm{MgFeMnO}_{4}$ indeed forms a cubic spinel structure with $\mathrm{Mg} / \mathrm{Fe} / \mathrm{Mn}$ disorder. Furthermore, macroscopic magnetization measurements show that the magnetic transition temperature is above room temperature (RT). A ferrimagnetic spin ordering is revealed below $T_{\mathrm{C}}=392 \mathrm{~K}$ by the combination of neutron powder diffraction (NPD) and muon spin rotation/ relaxation $\left(\mu^{+} \mathrm{SR}\right)$ measurements. These results provide a deeper understanding of the fundamental properties of $\mathrm{MgFeMnO}_{4}$ along with potential improvement of related battery devices.

\section{EXPERIMENTAL METHODS}

Materials Synthesis. Stoichiometric amounts of $\mathrm{Mn}_{2} \mathrm{O}_{3}$ (Kishida Chemicals, $99 \%$ purity), $\mathrm{Fe}_{2} \mathrm{O}_{3}$ (Kishida Chemicals, $98 \%$ purity), and $\mathrm{MgO}$ (99.9\%, Wako pure chemical) powders were weighted in a molar ratio of 1:1:1. The powders were mixed by using a planetary ball mill to attain a homogeneous mixture. The powder was pelletized and calcined at $800{ }^{\circ} \mathrm{C}$ for $5 \mathrm{~h}$ in air. Quantification of the chemical composition of the as-prepared $\mathrm{MgFeMnO}_{4}$ was assessed by using inductively coupled plasma absorption electron spectroscopy (ICPAES) on a Shimadzu ICPS-8100 instrument.

X-ray and Neutron Powder Diffraction. X-ray powder diffraction (XRPD) measurements of the as-synthesized powders were performed in Bragg-Brentano mode by using a diffractometer (Bruker D2 ADVANCE) at a $2 \theta$ range of $5^{\circ}-90^{\circ}$ using a step size of $0.01^{\circ}$. The wavelength was set at $\mathrm{Cu} \mathrm{K} \alpha$, and measurements were performed at room temperature. Neutron powder diffraction (NPD) was performed on the D2B instrument (ILL, Grenoble) using the selected neutron wavelength $\lambda=1.595 \AA$. Data were collected at two temperatures $T=300$ and $500 \mathrm{~K}$. The measurement at $500 \mathrm{~K}$ was performed by using a dedicated furnace. Symmetry analysis and Rietveld refinements for both X-ray and neutron diffraction patterns were performed with tools from the FULLPROF software suite ${ }^{18}$ and the Bilbao Crystallographic Server. ${ }^{19}$

Electron Microscopy. The morphology of the materials was analyzed by a field-emission scanning electron microscope (JSM6510LA). High-resolution transmission electron microscopy (HRTEM) images of pristine $\mathrm{MgFeMnO}_{4}$ were obtained on a TITAN80-300F at an accelerated voltage of $200 \mathrm{kV}$. Simulations of the HRTEM images were performed with the JEMS 31(PECD) software.

Magnetization Measurements. Bulk magnetic (DC) susceptibility measurements as a function of temperature were performed with a Quantum Design superconducting quantum interference device magnetometer (MPMS) upon warming in a temperature range from 5 to $400 \mathrm{~K}$. Data were collected in both zero-field-cooled (ZFC) and field-cooled (FC) protocols using an external magnetic field $H=100$ Oe. Magnetization data as a function of field were collected up to $\pm 10.0 \mathrm{kOe}$ after zero field cooling. For the data analysis, the coercive field $\left(H_{\mathrm{C}}\right)$ is defined as $H_{\mathrm{C}}=\left(H_{\mathrm{C}}^{+}+H_{\mathrm{C}}^{-}\right) / 2\left(I\left(H_{\mathrm{C}}^{-}-H_{\mathrm{C}}^{+}\right) \mid / 2\right)$, where the coercivity at the positive $\left(H_{\mathrm{C}}^{+}\right)$and negative $\left(H_{\mathrm{C}}^{-}\right)$fields has been determined by linear interpolation of the data points close to zero magnetization. In addition, the exchange bias field $\left(H_{\mathrm{EB}}\right)$ was extracted according to the following relation: $H_{\mathrm{EB}}=\left(\left|H_{\mathrm{C}}^{-}+H_{\mathrm{C}}^{+}\right|\right) / 2$. 
Mössbauer Spectroscopy. ${ }^{57} \mathrm{Fe}$ Mössbauer experiments were performed at $298 \mathrm{~K}$ by using a conventional Mössbauer spectrometer. About $100 \mathrm{mg}$ of sample was mixed with boron nitride and pressed into pellets (having a diameter of $10 \mathrm{~mm}$ ) for Mössbauer transmission experiments. $\alpha$-Fe was used for velocity calibration. The Mössbauer spectrum was fitted by using standard data analysis software (Origin Pro, version 2020b) by adding multiple Lorentzian peak functions. Also pseudo-Voigt functions were tested, yielding very similar results (see the Supporting Information, Figure S2 and Table S2). Finally, data were fitted by using the specialized MossWinn program. ${ }^{20,21}$

Muon Spin Rotation and Relaxation ( $\left.\mu^{+} S R\right)$ Measurements. $\mu^{+} S R$ experiments were performed by using the general purpose surface (GPS)-muon instrument ${ }^{22}$ at the Swiss Muon Source $(\mathrm{S} \mu \mathrm{S})$ of PSI in Switzerland. This spectrometer has a time resolution of $160 \mathrm{ps,}$ which corresponds to an upper theoretical cutoff frequency of 6250 $\mathrm{MHz}$. More realistically and experimentally proven the GPS spectrometer can resolve muon spin rotation frequencies up to $1842 \mathrm{MHz}^{22}$ Considering the gyromagneic ratio of the muon $\gamma_{\mu}=$ $135.5 \mathrm{MHz} / \mathrm{T}$, this means that the highest detectable internal field at the muon stopping site is approximately $1842 / 135.5 \approx 13.6 \mathrm{~T}$. The temperature range for the current experiments, $2-300 \mathrm{~K}$, was obtained by using a liquid-He flow-type horizontal cryostat. The powder sample $(m \approx 0.5 \mathrm{~g})$ was placed in an envelope made of very thin $(<50 \mu \mathrm{m})$ Al-coated Mylar tape covering a surface area of $1 \times 1$ $\mathrm{cm}^{2}$. To minimize the background signal, the envelope was attached to a fork-type $\mathrm{Cu}$ sample holder by a single layer of the Mylar tape. The sample holder was attached to a sample stick and inserted into the cryostat. The measurements above room temperature (RT) were performed by using a closed-cycle refrigerator (CCR). For such measurement the powder sample was pressed into a pellet that was attached onto a Cu-plate sample holder by Al-coated Mylar tape and secured by a thin $\mathrm{Cu}$ wire. For both setups, $\mu^{+} \mathrm{SR}$ time spectra were collected by using the zero-field (ZF) and weak transverse-field (wTF $=50 \mathrm{Oe}$ ) protocols. One of the main advantages of the $\mu^{+} \mathrm{SR}$ technique is that magnetic spin order can be studied in a true ZF environment. Further details regarding the experimental techniques and setups are provided in ref 23 . The musrfit ${ }^{24}$ software package was used to fit and analyze the $\mu^{+}$SR data.

\section{RESULTS}

Fundamental Characterization. XRPD was first performed to check the quality of the sample at room temperature. It is known that the stoichiometry of magnesium spinel oxides is sensitive to the heat treatments performed during the synthesis process. $^{25}$ The composition ratio of $\mathrm{MgFeMnO}_{4}$ was carefully examined also by using ICP-AES (Table S1). The synthesized $\mathrm{MgFeMnO}_{4}$ was confirmed to have the stoichiometric composition within the experimental accuracy. The concentration of $\mathrm{Mg}$ was found to be only slightly lower than that of $\mathrm{Fe}$ and $\mathrm{Mn}$, probably related to the evaporation of $\mathrm{Mg}$ during the heat treatment process. The detailed analysis of the XRPD data is described in the next section, together with the neutron diffraction results.

The temperature dependencies of the DC magnetic susceptibility, $\chi(T)$, for $\mathrm{MgFeMnO}_{4}$ by using both $\mathrm{ZFC}$ and FC protocols (measured upon warming) are shown in Figure 1a,b. As seen, the ZFC and FC magnetizations bifurcate at $T \approx$ $370 \mathrm{~K}$ and show a broad peak in the ZFC curve with a maximum at $T_{\max } \approx 330 \mathrm{~K}$. Such behavior supports the existence of antiferromagnetic and/or ferrimagnetic spin clusters. $^{26,27}$ When the sample is measured during the FC process, more ferro- or ferrimagnetic clusters are aligned along the field direction, which causes a large separation between FC and $\mathrm{ZFC} .{ }^{26} \mathrm{FC}$ susceptibility reaches its maximum around $T \approx$ $20 \mathrm{~K}$ and then saturates, which indicates a ferromagnetic behavior.
The correlation between the magnetization and the strength of the external applied magnetic field strength ( $M$ vs $H$ plots) at different temperatures is shown in Figure 1c,d. Hysteresis loops were observed at both $T=298 \mathrm{~K}$ and $T=5 \mathrm{~K} . M$ increases steeply for low fields, then rises relatively slowly, and does not fully saturate up until $H=10 \mathrm{kOe}$. The hysteresis curves of $\mathrm{MgFeMnO}_{4}$ show a soft ferromagnetic behavior at both temperatures. The coercivity $\left(H_{\mathrm{C}}\right)$ and retentivity $\left(M_{\mathrm{r}}\right)$ values at the two different temperatures are summarized in Table 1 . The coercivity is the magnitude of the field that must

Table 1. Results from the Magnetization vs Magnetic Field Measurements (See Figure 1c,d) ${ }^{a}$

\begin{tabular}{lll}
\multicolumn{1}{c}{ magnetic parameters } & $5 \mathrm{~K}$ & $298 \mathrm{~K}$ \\
magnetization $\left[M_{\mathrm{s}}\right]\left(\mu_{\mathrm{B}}\right)$ & 2.258 & 0.928 \\
coercivity $\left[H_{\mathrm{C}}\right](\mathrm{Oe})$ & 479.4 & 69.70 \\
retentivity $\left[M_{\mathrm{r}}\right]\left(\mu_{\mathrm{B}}\right)$ & 1.185 & 0.184 \\
squareness $[r]$ & 0.52 & 0.20 \\
exchange bias field $\left[H_{\mathrm{EB}}\right](\mathrm{Oe})$ & 9.740 & 37.23
\end{tabular}

${ }^{a_{T}}$ The table lists the saturation magnetization $\left(M_{\mathrm{s}}\right)$, coercivity $\left(H_{\mathrm{C}}\right)$, retentivity $\left(M_{\mathrm{r}}\right)$, squareness ratio/remanence ratio $(r)$, and the exchange bias field $\left(H_{\mathrm{EB}}\right)$ of $\mathrm{MgFeMnO}_{4}$ at $T=5 \mathrm{~K}$ and $T=298 \mathrm{~K}$.

be applied in the negative direction to bring the magnetization of the sample back to zero. These magnetic properties at RT are very similar to the features of the ferrimagnetic parent compound $\mathrm{MgFe}_{2} \mathrm{O}_{4}$. In contrast, the other parent compound $\mathrm{MgMn}_{2} \mathrm{O}_{4}$ displayed an antiferromagnetic spin order. ${ }^{28}$

Figure 2 shows the Mössbauer spectrum of $\mathrm{MgFeMnO}_{4}$ recorded at RT. The sextet splitting of the Mössbauer

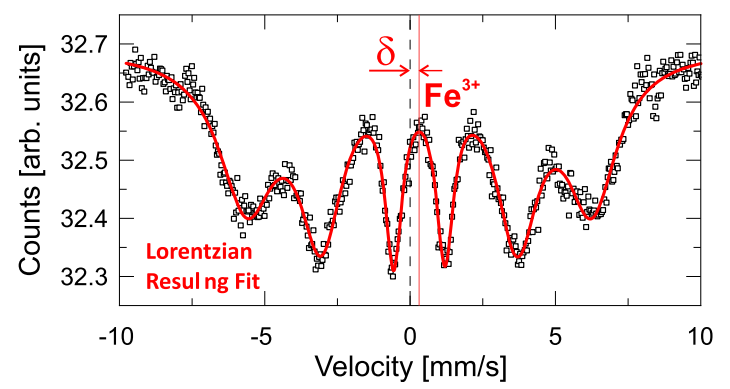

Figure 2. Mössbauer spectrum of $\mathrm{MgFeMnO}_{4}$ recorded at $\mathrm{T}=298 \mathrm{~K}$. Experimental data (black symbols) are fitted by using Lorentzian peak functions. The clear $\mathrm{Fe}^{3+}$ valence state is visible from the isomer shift $\delta=0.31 \mathrm{~mm} / \mathrm{s}^{26,29,30}$

spectrum indicates that $\mathrm{MgFeMnO}_{4}$ is in a magnetically ordered state ${ }^{31}$ at RT. This is consistent with the fact that the current Mössbauer data are clearly acquired in the spinordered phase of $\mathrm{MgFeMnO}_{4}$, as seen from, for example, the magnetization measurements in Figure 1. By using multiple Lorentzian peak functions, we can fit the data well using six components. The resulting peak positions and full width at half-maximum (FWHM) along with the respective peak splits, $\Delta_{i-j}$, are detailed in Table S2. First of all, it is clear that the peak shapes are broadened. This could be due to several reasons including multiple $\mathrm{Fe}$ sites in the lattice (see below for structure determination) along with several additional effects, for example, the particle size, superparamagnetism, spin glass, or spin cluster systems. Here, the particle size of the present sample was determined to be approximately $50-200 \mathrm{~nm}$, as extracted from SEM measurements (images are shown in 
Figure S1). It should be noted that nanoparticles often yield a superparamagnetic contribution. Additional broadening may also stem from a wide magnetic hyperfine distribution as also observed in $\mathrm{MgFe}_{2} \mathrm{O}_{4} \cdot{ }^{14}$

An isomer shift $\delta=0.31 \mathrm{~mm} / \mathrm{s}$ was extracted from our data by using the MossWin fitting software. ${ }^{20}$ Such a value of $\delta$ supports that an iron valence $\mathrm{Fe}^{3+}$ rather than $\mathrm{Fe}^{2+}$ is present in $\mathrm{MgFeMnO}_{4}{ }^{26,29,30}$ In addition, a mixed valence case containing both $\mathrm{Fe}^{2+}$ and $\mathrm{Fe}^{3+}$ would cause an asymmetric peak shape (Figure 2a), which is not (clearly) visible. Moreover, the quadrupole splitting (QS) was not discernible for the magnetically split spectrum; ${ }^{31}$ thus, the spin state of $\mathrm{Fe}$ cannot be determined from our Mössbauer data. It is noteworthy that $\mathrm{Fe}$ is normally stable in the high-spin (HS) state; on the other hand, low-spin and intermediate-spin states can be induced by applying high pressure. ${ }^{29}$ The parent compound, $\mathrm{MgFe}_{2} \mathrm{O}_{4}$, is indeed reported to have high-spin $\mathrm{Fe}^{3+} \cdot{ }^{32}$ In similarity to $\mathrm{MgFeMnO}_{4}$, the $\mathrm{QS}$ for $\mathrm{MgFe}_{2} \mathrm{O}_{4}$ was also not resolved in the magnetically ordered state but could be observed in the paramagnetic phase above the magnetic transition temperature. Even though we did not have the possibility to measure $\mathrm{MgFeMnO}_{4}$ in its paramagnetic state, it is very reasonable to surmise that $\mathrm{Fe}^{3+}$ also in $\mathrm{MgFeMnO}_{4}$ would adopt the high-spin state at ambient pressure.

The magnetism of spinel ferrites is known to be strongly related to the cation distribution between the $A$ and $B$ sites. For instance, Osmond et al. claimed that the disorder of the magnetic cation in both $A$ and $B$ sites induces ferrimagnetic interactions. ${ }^{33}$ However, as De Grave et al. mentioned in ref 14 , it is impossible to determine the $\mathrm{Fe}^{3+}$ cation distribution among $A$ and $B$ sites of a spinel lattice from using only zerofield Mössbauer spectroscopy. To investigate the details of the cation distribution, a careful crystal structure analysis was performed by combining both X-ray and neutron powder diffraction measurements.

Crystal Structure Determination. The crystal structure of $\mathrm{MgFeMnO}_{4}$ was investigated by using both XRPD and NPD techniques. The neutron scattering technique was absolutely necessary because of its higher sensitivity to oxygen as well as greater $\mathrm{Mn} / \mathrm{Fe}$ contrast, in comparison to XRPD. The structural analysis of $\mathrm{MgFeMnO}_{4}$ started from the reported space group, unit cell, and atomic coordinates of cubic spinel $\mathrm{MgFe}_{2} \mathrm{O}_{4} \cdot{ }^{34,35}$ The XRPD and NPD patterns of $\mathrm{MgFeMnO}_{4}$ are indexed perfectly by using a cubic crystal structure (space group $F d \overline{3} m$, No. 227) with $a \sim 8.43 \AA$. The space group and cell parameters are also confirmed by electron diffraction recorded at RT (see Figure S1c,d). To avoid the influence of magnetic Bragg peaks, the NPD pattern recorded at $T=500 \mathrm{~K}$ (i.e., above magnetic transition temperature) was used to initially determine the crystal structure. The crystallographic parameters obtained from the refinement performed on the NPD data $(T=300 \mathrm{~K}$ and $T=500 \mathrm{~K})$ and the corresponding interatomic distances are presented in Table 2. Figure 3 shows the resulting XRPD and NPD patterns along with the calculated patterns. The crystal structure obtained from the calculated patterns is displayed in Figure 4. The values of the lattice parameter of the cubic structure, determined in this study, are $8.4290 \AA$ at $T=500 \mathrm{~K}$ and $8.4116 \AA$ at $T=300 \mathrm{~K}$. The values are comparable to the reported cell parameter of $\mathrm{MgFe}_{2} \mathrm{O}_{4}$ studied by synchrotron X-ray diffraction (at $500 \mathrm{~K}: a$ $=8.437 \AA$; at $300 \mathrm{~K}: a=8.4208 \AA) .^{36}$

No signs of impurity phase peaks were observed in either XRPD or NPD patterns, indicating that the synthesized sample
Table 2. Structural Parameters of $\mathrm{MgFeMnO}_{4}$ at $T=500 \mathrm{~K}$ and $T=300 \mathrm{~K}$ from Rietveld Refinement of Neutron Powder Diffraction (NPD) Data ${ }^{a}$

\begin{tabular}{lll}
\multicolumn{1}{c}{$T=500 \mathrm{~K}$} & $T=300 \mathrm{~K}$ \\
$a(\AA)$ & \multicolumn{1}{c}{ structural parameters } \\
$V\left(\AA^{3}\right)$ & $8.4290(1)$ & $8.4116(1)$ \\
$x$ & $598.9(1)$ & $595.2(1)$ \\
$O_{(A)}(\mathrm{Mg} / \mathrm{Fe} / \mathrm{Mn})$ & $0.2587(1)$ & $0.2587(1)$ \\
$B_{\text {iso }(A)}\left(\AA^{2}\right)$ & $0.325(2) / 0.241(2) / 0.434(2)$ & \\
$O_{[B]}(\mathrm{Mg} / \mathrm{Fe} / \mathrm{Mn})$ & $0.350(2) / 0.518(2) / 0.133(2)$ & $0.63(2)$ \\
$B_{\text {iso }[B]}\left(\AA^{2}\right)$ & $0.73(3)$ & $0.43(3)$ \\
$B_{\text {iso oxygen }}\left(\AA^{2}\right)$ & $1.30(1)$ & $1.08(1)$ \\
$\chi^{2}(\AA)$ & 3.05 & 3.93 \\
$R_{\text {Bragg }}(\AA)$ & 2.52 & 2.24 \\
$A-\mathrm{O}(\times 4)$ & selected interatomic distances $(\AA)$ & \\
$B-\mathrm{O}(\times 6)$ & $1.951(1)$ & $1.948(1)$ \\
$A-A$ & $2.037(1)$ & $2.033(1)$ \\
$B-B$ & $3.650(1)$ & $3.642(1)$ \\
$A-B$ & $2.980(1)$ & $3.974(1)$ \\
$T-B$ & $3.494(1)$ &
\end{tabular}

${ }^{a_{T}}$ The space group Is $F \bar{d} m$ and cations occupy Wyckoff positions $8 a$ [tetrahedral sites $(A)]$ and $16 d$ (octahedral sites $[B]$ ). The fractional coordinates of $(A)$ and $[B]$ positions are $(1 / 8,1 / 8,1 / 8)$ and $(1 / 2,1 /$ $2,1 / 2)$, respectively. Oxygen atoms occupy Wyckoff position $32 e$ with fractional coordinates $(x, x, x)$. The isotropic thermal factors $\left(B_{\text {iso }}\right)$ as well as fractional occupancies $\left(O_{(A) /[B]}\right)$ are given for the different sites. Finally, the goodness of fit is also detailed.

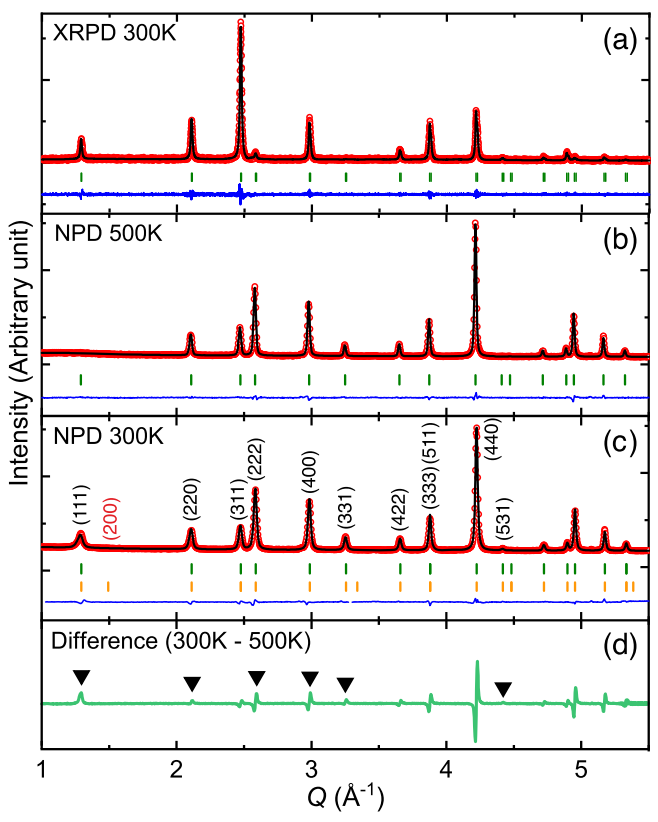

Figure 3. Rietveld refinements of $\mathrm{MgFeMnO}_{4}$ : (a) XRPD data at $T=$ $300 \mathrm{~K}\left(R_{\text {Bragg }}=3.8 \%\right.$ and global $\left.\chi^{2}=1.6\right)$, (b) NPD data at $T=500 \mathrm{~K}$ $\left(R_{\text {Bragg }}=2.5 \%\right.$ and global $\left.\chi^{2}=3.1\right)$, and $(c)$ NPD data at $T=300 \mathrm{~K}$ $\left(R_{\text {Bragg }}=2.2 \%, R_{\text {Braggmag }}=17 \%\right.$ and global $\left.\chi^{2}=3.9\right)$. Experimental data: open red circles; calculated profile: solid black line; allowed Bragg reflections: vertical marks. The difference between the experimental and calculated profiles is displayed at the bottom of each graph as a blue line. (d) Difference between NPD data between recorded at $T=300 \mathrm{~K}$ and $T=500 \mathrm{~K}$. Black triangles highlight additional Bragg intensities due to the magnetic ordering.

is a single high-quality phase. The structure is a typical spinel structure, and the three cations $(\mathrm{Mg}, \mathrm{Fe}$, and $\mathrm{Mn})$ are 

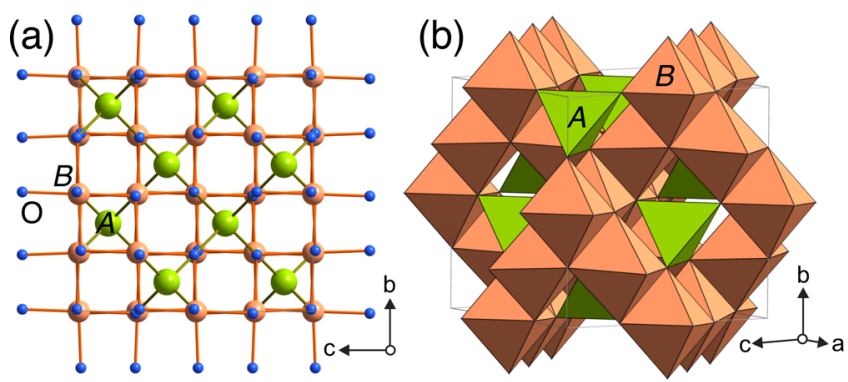

Figure 4. Crystal structure of the cubic $(F d \overline{3} m)$ spinel structure (a) viewed along (100) with cation-oxygen bonds and (b) polyhedra representation. Tetrahedral $A$ sites and octahedral $B$ site are displayed in green and orange, respectively. The refined cation occupancies on respective site of $\mathrm{MgFeMnO}_{4}$ are $A(\mathrm{Mg} / \mathrm{Fe} / \mathrm{Mn}=33 / 24 / 43 \%)$ and $B(\mathrm{Mg} / \mathrm{Fe} / \mathrm{Mn}=35 / 52 / 13 \%)$.

distributed in both tetrahedral and octahedral oxygen environments. The cation distribution was determined by careful refinements performed by using a step-by-step protocol. First, the ratio of $\mathrm{Mg}$ and $\mathrm{Fe} / \mathrm{Mn}$ was obtained from the refinement of the laboratory XRPD pattern, and the result was then used as starting value for the first step of the NPD pattern fit process. The resulting cation distributions are as follows: the tetrahedral site is occupied by $\mathrm{Mg}: \mathrm{Fe}: \mathrm{Mn}=33 \%: 24 \%: 43 \%$, whereas the octahedral site by $\mathrm{Mg}: \mathrm{Fe}: \mathrm{Mn}=35 \%: 52 \%: 13 \%$.

Cation disorder within spinel ferrites have been reported to change as a function of temperature. ${ }^{25,36}$ However, for the current $\mathrm{MgFeMnO}_{4}$ compound, the nuclear Bragg peaks at $\mathrm{T}=$ $300 \mathrm{~K}$ and $T=500 \mathrm{~K}$ of NPD patterns do not show any significant differences, which implies that the cation distribution remains the same in the entire temperature range. This is consistent with a previous report on $\mathrm{MgFe}_{2} \mathrm{O}_{4}{ }^{36}$ which suggests that the cation distribution remains unchanged in the temperature range $\mathrm{RT}$ to $T=773 \mathrm{~K}$. The subsequent magnetic structure determination for $\mathrm{MgFeMnO}_{4}$ was therefore performed under the assumption that the cation distribution does not change between $T=300 \mathrm{~K}$ and $T=$ $500 \mathrm{~K}$.

Magnetic Structure Determination. From the magnetic susceptibility curve (Figure 1a), either ferromagnetic or ferrimagnetic interactions are expected to govern the magnetic ordering of $\mathrm{MgFeMnO}_{4}$ below the magnetic transition temperature. Comparing the two neutron diffraction patterns recorded above and below the magnetic transition temperature, the appearance of new magnetic Bragg peaks (Figure $3 b-d)$ clearly reveals the presence of long-range magnetic ordering. There is an enhancement of the intensity of the Bragg peaks indexed (111), (220), (222), (400), (331), and (531) due to the magnetic contributions for $T<T_{\mathrm{C}}$ (highlighted by black triangles in Figure 3d). All the magnetic Bragg peaks can be indexed with a commensurate propagation vector $k=(000)$. This means that the magnetic unit cell is the same as the crystallographic unit cell, having identical symmetry relations. Therefore, the nuclear and magnetic contributions to the Bragg reflections occur at the same scattering angles (i.e., nuclear and magnetic contributions of Bragg reflections are overlapping).

For the magnetic spin analysis, each model was refined to convergence, and the best result was selected based on agreement factors and stability of the refinement. The occupancy of cations on tetrahedral and octahedral sites obtained from the earlier refinements were kept fixed, and all other parameters were varied freely. Figure $3 \mathrm{c}$ shows a neutron diffraction pattern containing both nuclear and magnetic contributions. The solid line is the resulting refinement based on two-phase analysis, showing a satisfactory agreement between the observed and calculated profiles. Because there is no magnetic Bragg peak observed at the position of (200) (indexed and highlighted in red in Figure 3c), the spin orientation can be concluded to have only one magnetic component (i.e., as described in Figure 5). The resulting

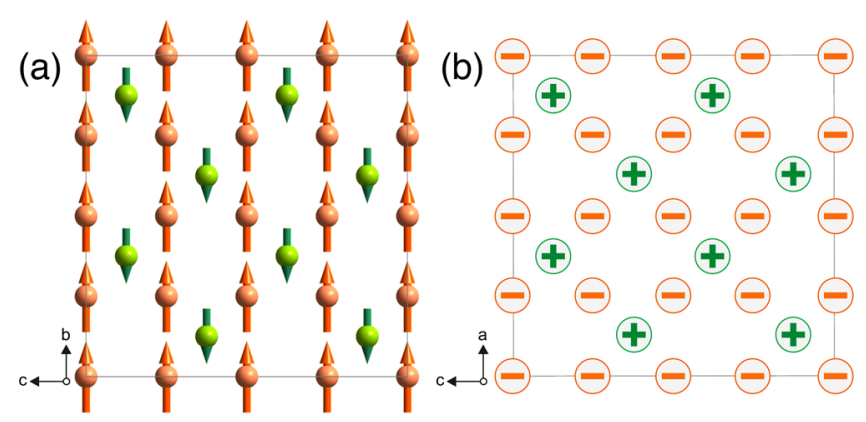

Figure 5. Refined ferrimagnetic spin structure model of $\mathrm{MgFeMnO}_{4}$ at $300 \mathrm{~K}$. (a) Projection along (100) and (b) (010). As an example, the magnetic component is aligned in $\pm m_{y}$. In (b) the moments are along $b$ and symbolized by + or signs depending on their direction. The used color coding is coherent with Figure 4; i.e., tetrahedral $A$ sites and octahedral $B$ site are displayed in green and orange, respectively.

magnetic structure at $T=300 \mathrm{~K}$ having ordered magnetic moments of $A=1.57$ (5) and $B=1.22$ (4) $\mu_{\mathrm{B}}$. The spins of the $A$ and $B$ sites are found to align in a collinear but antiparallel fashion, hereby suggesting that the magnetic structure is ferrimagnetic. The resulting spin arrangement of $\mathrm{MgFeMnO}_{4}$ is shown schematically in Figure 5.

Muon Spin Relaxation $\left(\boldsymbol{\mu}^{+} \boldsymbol{S R}\right)$. We also performed positive muon spin rotation/relaxation $\left(\mu^{+} S R\right)$ measurements of $\mathrm{MgFeMnO}_{4}$ under weak transverse field ( $\mathrm{wTF}=50 \mathrm{Oe}$ ) (Figure 6) and zero field (ZF) (Figure 7) at temperatures between 2 and $470 \mathrm{~K}$. The wTF- $\mu^{+}$SR spectrum was fitted by using a combination of a slowly (exponentially) relaxing oscillatory (cosine) signal due to the externally applied magnetic field $\mathrm{wTF}=50 \mathrm{Oe}$ and a nonoscillating but exponentially relaxing ("mag") signal:

$$
\begin{aligned}
& A_{0} P_{\mathrm{TF}}(t)=A_{\mathrm{TF}} \cos \left(2 \pi \omega_{\mathrm{TF}} t+\phi_{\mathrm{TF}}\right) \exp \left(-\lambda_{\mathrm{TF}} t\right) \\
& +A_{\mathrm{mag}} \exp \left(-\lambda_{\mathrm{mag}} t\right)
\end{aligned}
$$

Here $P_{\mathrm{TF}}(t)$ is the muon spin polarization function, $A_{0}$ is the initial asymmetry, $A_{\mathrm{TF}}$ and $A_{\mathrm{mag}}$ are the asymmetries of the related polarization components, and $2 \pi \omega_{\mathrm{TF}}$ is the angular frequency of the Larmor precession (of the rotating muon spins) under the applied transverse field. In addition, $\lambda_{\mathrm{TF}}$ and $\lambda_{\text {mag }}$ are the exponential relaxation rates for the two components, and $\phi_{\mathrm{TF}}$ is the initial phase of the precessing signal. In particular, $A_{\mathrm{TF}}$ is the fraction of muons sensing the applied wTF $=50 \mathrm{Oe}$, while $A_{\mathrm{mag}}$ is the fraction of muons coupled to the internal field. Note that the experimental setup was changed at $300 \mathrm{~K}$ from low-temperature cryostat to hightemperature CCR. The time spectra at $300 \mathrm{~K}$ were collected by using both setups to record an overlapping data point. There is no precessing signal $\left(A_{\mathrm{TF}}=0\right)$ at $300 \mathrm{~K}$ when using the cryostat, but $A_{\mathrm{TF}}=0.06$ is observed when the setup was 

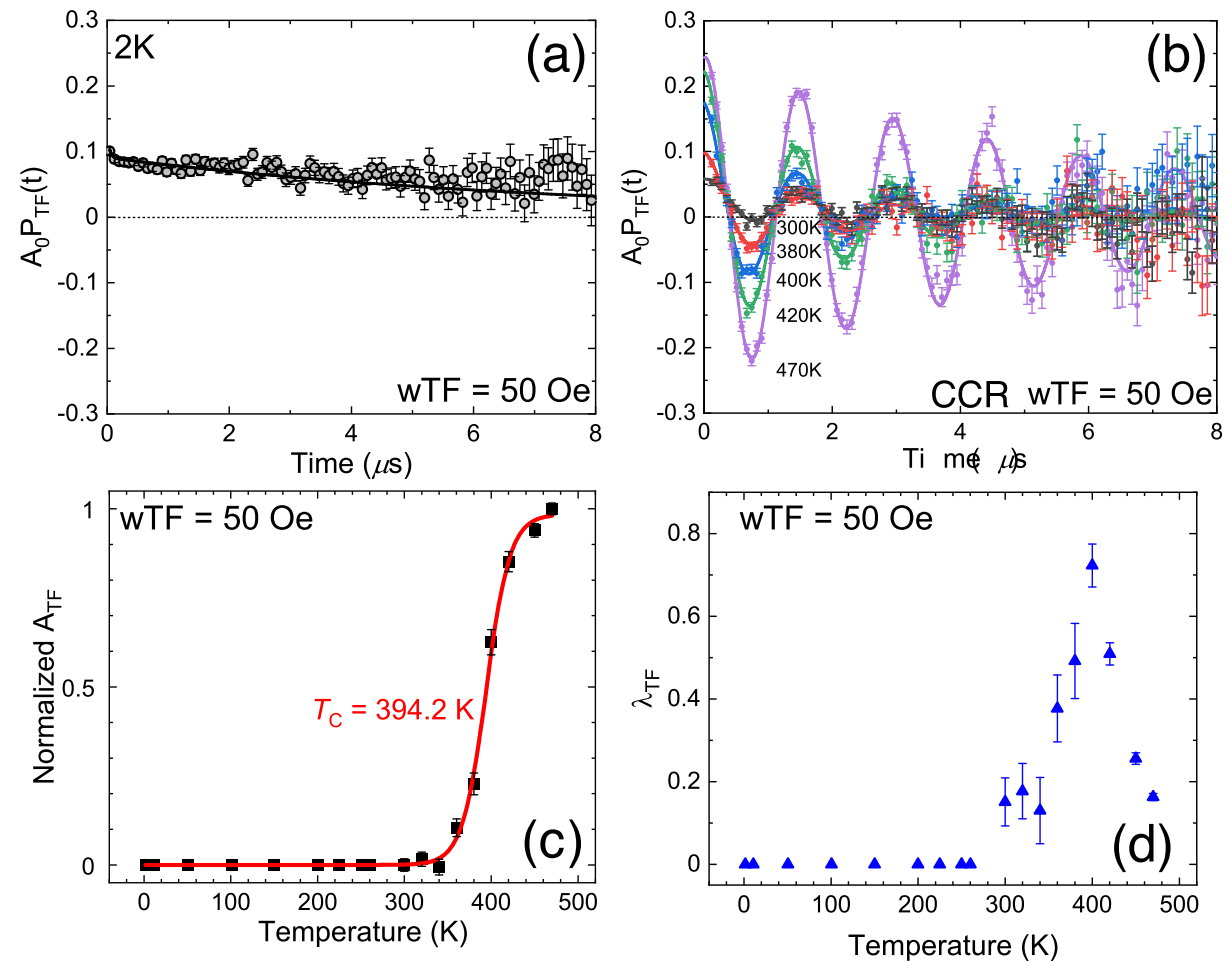

Figure 6. (a) $\mu^{+} \mathrm{SR}$ time spectra of $\mathrm{MgFeMnO}_{4}$ at $T=2 \mathrm{~K}$ (in cryostat) and (b) temperature dependence $\mu^{+} \mathrm{SR}$ time spectra (at 300, 380, 400, 420, and $470 \mathrm{~K}$ in CCR) under a weak-transverse field, wTF $=50$ Oe. The corresponding fits using eq 2 are shown as solid lines. For clarity, $A_{0}$ is the initial asymmetry and $P_{\mathrm{TF}}(t)$ is the muon spin polarization function. (c) Temperature dependence of the normalized wTF asymmetry $A_{\mathrm{TF}}$ of $\mathrm{MgFeMnO}_{4}$, obtained from fits to the wTF $\mu^{+}$SR time spectra using eq 2. In addition, the sigmoid fit (red solid line) yields the magnetic transition temperature, $T_{\mathrm{C}}=394.2 \mathrm{~K}$. (d) wTF relaxation rate $\left(\lambda_{\mathrm{TF}}\right)$ displaying a clear peak in the vicinity of the magnetic phase transition.
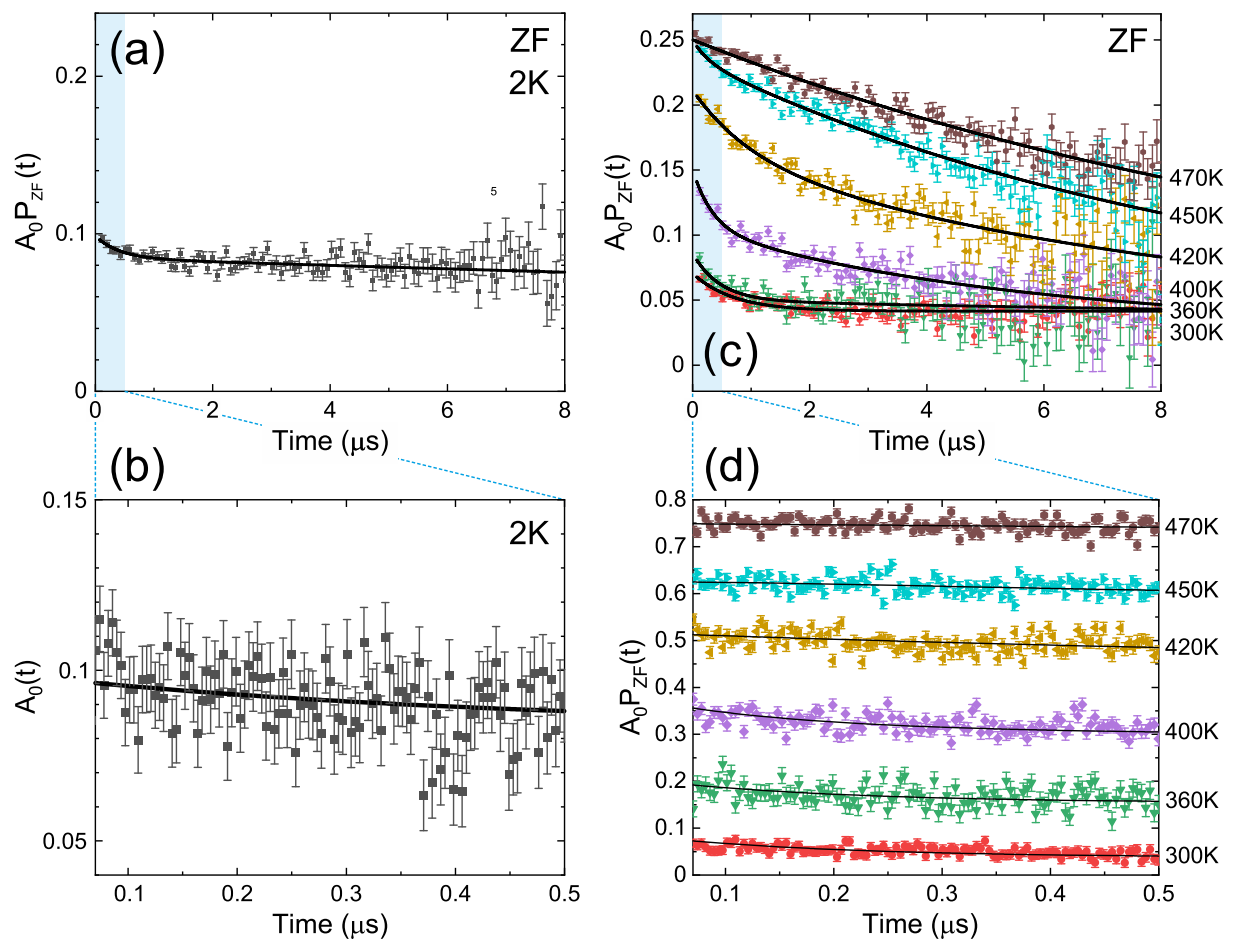

Figure 7. (a) ZF- $\mu^{+} \mathrm{SR}$ time spectra of $\mathrm{MgFeMnO}_{4}$ at $T=2 \mathrm{~K}$ shown for the complete time domain. A shaded blue rectangle is shown in (b) where a zoomed-in view of the shorter time domain emphasizes the absence of any clear oscillations along with a missing asymmetry fraction. (c) Temperature-dependent ZF- $\mu^{+}$SR spectra for $\mathrm{MgFeMnO}_{4}$ in the long time domain up to $8 \mu \mathrm{s}$. A shaded blue rectangle is shown in (d) with a zoomed-in view of the early time domain up to $0.2 \mu \mathrm{s}$. For $(\mathrm{c}, \mathrm{d})$ each spectrum is offset along the $y$-axis by 0.1 for clarity of display. Solid lines are the best fits to the data using eq 2 . 
changed to the CCR. The additional asymmetry stems from muons hitting the $\mathrm{Cu}$ sample holder, that is, a courtesy of the higher background signal of the CCR setup. The wTF signal shown in Figure $6 \mathrm{~b}$ was measured by using CCR.

Because the applied field is weak relative to the internal field (when the sample is magnetically ordered), the fraction of normalized asymmetry $\left(N_{\mathrm{ATF}}=A_{\mathrm{TF}} / \max \left[A_{\mathrm{TF}}\right]\right)$ with background subtracted then corresponds to the paramagnetic (PM) fraction of the sample. Therefore, the temperature dependence of $A_{\mathrm{TF}}(T)$ can be considered as the evolution of the magnetic state in the sample. By plotting $N_{\text {ATF }}$ vs $T$ (see Figure $6 \mathrm{c}$ ), we can fit the trend of the asymmetry with a sigmoid function. From such fit, the transition temperature $T_{\mathrm{C}}=394.2(12) \mathrm{K}$ is defined as the middle point of the fitting curve, where $N_{\mathrm{ATF}}=$ 0.5 . Such a value of $T_{\mathrm{C}}$ is in excellent agreement with our other experimental results mentioned previously. From the wTF spectra collected below $T_{\mathrm{C}}$ it is clear that the entire volume of the sample enters into a long-range magnetic order (since $A_{\mathrm{TF}}$ $=0$ at low temperatures as shown in Figure 6a). This is a clear indication that the investigated sample is homogeneous and of high quality. The unique possibility to accurately determine the magnetically ordered volume fraction of a sample is a powerful capability of the $\mu^{+} S R$ technique. Finally, the temperature dependence of the wTF relaxation rate $\left(\lambda_{\mathrm{TF}}\right)$ is shown in Figure 6d, displaying a clear peak in the vicinity of the magnetic phase transition. Here the strong increase in $\lambda_{\mathrm{TF}}$ indicates the expected and drastic change in the spin dynamics associated with the spin order.

Figure $7 \mathrm{a}$ shows $\mathrm{ZF}-\mu^{+} \mathrm{SR}$ time spectra recorded at the lowest experimental temperature, $T=2 \mathrm{~K}$. It is apparent that no oscillation signal is present even in the shortest time range (see the inset of Figure 7a). However, we clearly observe that the initial asymmetry at time zero is only $A_{0} \approx 0.1$ instead of the expected $\sim 0.24$ (determined by the experimental setup). This so-called "missing fraction" is directly related to the ferrimagnetic order and will be further discussed below. In fact, no oscillatory signal was observed in the entire experimental temperature range $(T=2-470 \mathrm{~K}$, see also Figure $7 \mathrm{~b}, \mathrm{c})$. Consequently, the ZF spectra were fitted by three exponentially relaxing nonoscillatory components. Here one of such signals is due to the paramagnetic background (BG) signal coming from the $\mathrm{Cu}$ holder in the high-temperature wTF CCR measurements (i.e., such component was only used for the data recorded above $300 \mathrm{~K}$, where $A_{\mathrm{BG}}$ was fixed to 0.0284). The resulting $\mathrm{ZF}$ fit function is described by

$$
\begin{aligned}
& A_{0} P_{\mathrm{ZF}}(t)=A_{\text {fast }} \exp \left(-\lambda_{\text {fast }} t\right)+A \exp \left(-\lambda_{\text {tail }} t\right) \\
& \quad+A_{\mathrm{BG}} \exp \left(-\lambda_{\mathrm{BG}} t\right)
\end{aligned}
$$

where $A_{0}$ is the initial asymmetry, $A_{\text {fast }} A_{\text {tail }}$, and $A_{\mathrm{BG}}$ are the asymmetries associated with each signal, and $\lambda_{\text {fast }} \lambda_{\text {tail }}$, and $\lambda_{\mathrm{BG}}$ are the exponential relaxation rates of each signal. It is clearly shown in Figure 7 that both short $(t<0.2 \mu \mathrm{s})$ and long $(t$ up to $8 \mu \mathrm{s}$ ) time spectra were well fitted by using eq 2 . At $T=2 \mathrm{~K}$ $\left(A_{\mathrm{BG}}=0\right), A_{\text {tail }}$ is about 0.08 , which is coherent with the expected $1 / 3$ (i.e., $0.24-0.0284 / 3$ ) powder average tail component (local field oriented parallel to the muon spin). This further supports the wTF results showing that the volume fraction of the magnetic phase is effectively $100 \%$ below $T_{\mathrm{C}}$. $A_{\text {fast}}$, on the other hand, corresponds to internal field components that are perpendicular to the initial muon spin. This fraction should correspond to about $2 / 3$ of the local fields in an ideal powder that is magnetically ordered. In other words, an asymmetry $2 / 3 A_{0}=0.16$ is expected if the depolarization is within the time resolution of the experimental setup. In this case we clearly have a missing fraction since we only find that $A_{\text {fast }} \approx 0.03$ at $T=2 \mathrm{~K}$.

The fitting parameters of the ZF- $\mu^{+}$SR time spectra above $300 \mathrm{~K}$ are summarized in Figure 8. It is noted that the

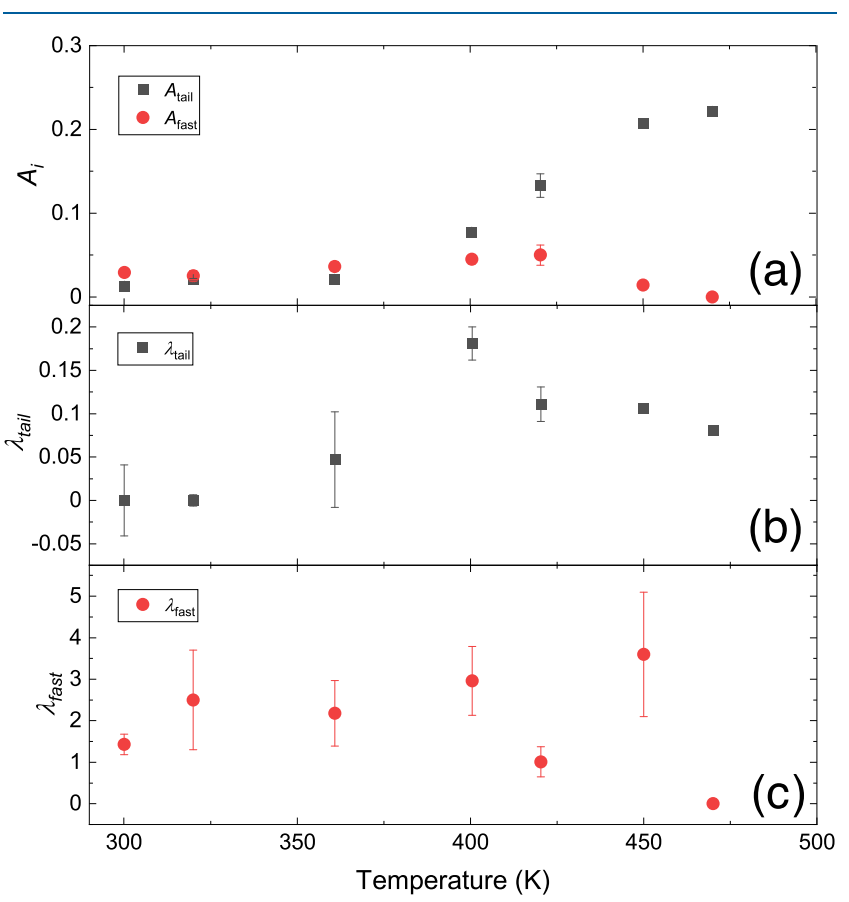

Figure 8. Temperature dependencies of the $\mathrm{ZF}-\mu^{+} \mathrm{SR}$ fit parameters for $\mathrm{MgFeMnO}_{4}$ : (a) the asymmetries $\left(A_{\text {tail }}\right.$ and $\left.A_{\text {fast }}\right)$, (b) the relaxation rate of tail component $\left(\lambda_{\text {tail }}\right)$, and $(c)$ the relaxation rate of fast component $\left(\lambda_{\text {fast }}\right)$. The parameters were obtained by fitting the $\mathrm{ZF}-\mu^{+} \mathrm{SR}$ spectra for $T \geq 300 \mathrm{~K}$ via eq 2 .

paramagnetic (BG) signal from the high-temperature setup comes from the $\mathrm{Cu}$ sample holder. A previous $\mu^{+} \mathrm{SR}$ study of the $\mathrm{Cu}$ metal shows a small relaxation rate $\left(\lambda_{\mathrm{BG}} \sim 0.025\right)$ observed at $300 \mathrm{~K}$, and it decreases further by increasing the temperature. ${ }^{37}$ Because $A_{\mathrm{BG}}$ is also small, it is difficult to discern such minor relaxation contribution for our present data. Thus, we fixed $\lambda_{\mathrm{BG}}=0$, which is a good approximation for our purposes.

In the temperature dependence of $\lambda_{\text {tail }}$ a maximum is observed at $T_{\max }=400 \mathrm{~K}$. Such a maximum occurs close to the inflection point of $A_{\text {tail }}$ and is directly related to a critical slowing down of magnetic moments accompanied by the magnetic order ( $c f$. the coherent behavior of $\lambda_{\mathrm{TF}}$ above in Figure $6 \mathrm{~d}$ ). Such a magnetic ordering temperature is in good agreement with the one determined by the wTF- $\mu^{+} S R$ data, that is, $T_{\mathrm{C}}=394.2 \mathrm{~K}$. Below $T_{\mathrm{C}}$, the internal magnetic fields are strong enough to create a large field distribution at the muon site, that is, to produce a fast depolarization of the muon beam (faster than the time resolution of the current experimental setup).

This is why we see such a large "missing fraction" as mentioned above. The resulting internal fields at the muon stopping sites are so strong that the muon spin precession is simply too fast, and the oscillation occurs below $t \approx 0.1 \mu \mathrm{s}$, that is, faster than the time resolution of the GPS spectrometer. Such situation is often observed in $\mu^{+}$SR spectra recorded in $\mathrm{ZF}$ for materials with very large magnetic moments, for 
instance, ferro-/ferrimagnetic materials. ${ }^{38-40}$ Because the magnetic moments of each magnetic site in $\mathrm{MgFeMnO}_{4}$ are maximum $\sim 1.6 \mu_{\mathrm{B}}$, such a scenario is very reasonable. Hence, this is also in perfect agreement with the current NPD results that reveal a collinear ferrimagnetic ordering.

\section{DISCUSSION}

Cation Distribution and Electrochemical Performance. Magnesium is generally known to be in a divalent state at tetrahedral lattice sites, whereas $\mathrm{Fe}$ and $\mathrm{Mn}$ are usually trivalent in the octahedral sites. The trivalent state of $\mathrm{Fe}$ is confirmed by Mössbauer spectroscopy (Figure 2). Assuming that $\mathrm{Mg}$ is stable in divalent state and all $\mathrm{Fe}$ are in trivalent state, then all $\mathrm{Mn}$ ions also have to be in trivalent state. To ultimately confirm the accurate valence state of the cations, further experiments, such as X-ray absorption near-edge spectroscopy (XANES), are required. In fact, XANES studies of the very closely related $\mathrm{Mg}\left(\mathrm{Mn}_{0.4} \mathrm{Fe}_{0.6}\right)_{2} \mathrm{O}_{4}$ compound at the $\mathrm{Mn}$ and $\mathrm{Fe} \mathrm{K}$-edge have indeed shown that both $\mathrm{Mn}$ and $\mathrm{Fe}$ are mainly in the trivalent state rather than the divalent state. ${ }^{6}$ Consequently, it is very reasonable to expect that $\mathrm{MgFeMnO}_{4}$ also displays similar valence states. The trivalent state of $\mathrm{Mn}$ in octahedra site ( $\mathrm{HS} \mathrm{d}^{4}: \mathrm{t}_{2 \mathrm{~g}}^{3} \mathrm{e}_{\mathrm{g}}^{1}$ ) is a Jahn-Teller-active cation, which is the origin of the distorted spinel $\mathrm{MgMn}_{2} \mathrm{O}_{4}$. However, the non-Jahn-Teller cations $\left(\mathrm{Fe}^{3+}\right.$ with $\left.\mathrm{HS} \mathrm{d}^{5}: \mathrm{t}_{2 \mathrm{~g}}^{3} \mathrm{e}_{\mathrm{g}}^{2}\right)$ suppresses such distortion. Another interesting point is that both octahedral and tetrahedral $\mathrm{Mn}$ sites can have an orbital degree of freedom $\left(t_{2 g}+e_{g}\right.$ are not completely filled, and electrons can occupy both orbitals). Therefore, our current results concerning both $\mathrm{Mn}$ valence and site occupancy should also be of high interest for future applications, such as spintronics and ultimately quantum computing. ${ }^{41-43}$

The electrochemical properties of the $\mathrm{Mg}\left(\mathrm{Mn}_{1-x} \mathrm{Fe}_{x}\right)_{2} \mathrm{O}_{4}$ series of compounds have been studied by Han et al. ${ }^{6}$ It was shown that the redox processes mainly occur between $\mathrm{Mn}^{3+}$ and $\mathrm{Mn}^{2+}$. However, it is necessary to substitute more than $20 \%$ of $\mathrm{Mn}$ with Fe to stabilize the cubic crystal structure, which yields the best charge/discharge performance. In our current study of $\mathrm{MgFeMnO}_{4}$ (i.e., $x=0.5$ ), we do not present results from electrochemical characterization. However, we naturally expect similar (intermediate) performance as $x=0.4$ and $x=0.6$, which is reported in ref 6 . Such compositions showed much better electrochemical properties compared to the parent compounds, $\mathrm{MgFe}_{2} \mathrm{O}_{4}$ and $\mathrm{MgMn}_{2} \mathrm{O}_{4}$. Moreover, disorder between the tetrahedral and octahedral sites in spinel oxides is known to create "anti-site defects", resulting in physical, chemical, optical, and electrochemical properties, which are favorable for battery devices as well as other applications. ${ }^{16,44}$ This effect relates to the interesting selfsubstitution occurring between tetrahedral and octahedral sites. The normal spinel structure consists of divalent ions occupying the tetrahedral site and trivalent ions placed in the octahedral site. If a distribution of cations between the two sites is present, a mixed valence state becomes possible: for instance, if a trivalent cation is placed in the tetrahedral $A$ site. Then, to compensate for the trivalent valence, the compound became the electron producing donor state (n-type). On the other hand, if a divalent cation is substituted into the octahedral $B$ site, it will instead form a hole producing acceptor state (p-type) to compensate for the divalent valence. Most of the produced holes/electrons will be electrochemically compensated. However, based onthe cation occupancy determined by our diffraction studies, $\mathrm{MgFeMnO}_{4}$ could in fact be a p-type semiconductor. Normally, antisite defects tend to degrade the ion-transport performance by effectively blocking the diffusion paths. ${ }^{4-47}$ However, because of this interesting self-substitution phenomenon, the resulting cathode performance can instead be improved through its propensity toward semiconducting properties. ${ }^{44,48}$ In this study, an accurate cation distribution of $\mathrm{MgFeMnO}_{4}$ was determined by combining X-ray and neutron powder diffraction. From our results it is clear that such antisite defects are present in this series of materials.

Finally, $\mathrm{Mg}^{2+}$ is only located at the tetrahedral $A$ site in $\mathrm{MgMn}_{2} \mathrm{O}_{4} \cdot{ }^{15-17}$ On the other hand, $\mathrm{MgFe}_{2} \mathrm{O}_{4}$ shows a certain degree of antisite defects $(10-30 \%$ of $\mathrm{Mg}$ in the octahedral $B$ site $\left.^{36}\right)$. In the current case of $\mathrm{MgFeMnO}_{4}$, the mixture of manganese and iron in the spinel oxide clearly increases the amount of antisite defects. The same effect was observed in $\mathrm{Mg}\left(\mathrm{Mn}_{2-x} \mathrm{Co}_{x}\right) \mathrm{O}_{4} \cdot{ }^{16}$ Moreover, in the case of $\mathrm{Mg}\left(\mathrm{Mn}_{2-x} \mathrm{Co}_{x}\right)$ $\mathrm{O}_{4}$, Zhao et al. claimed that the antisite effect plays a critical role to improve the electrochemical performance. ${ }^{16}$ Our current results, together with the previously reported electrochemical performance studies, ${ }^{6}$ further support the critical role of the antisite defects for improved electrochemical behavior. Consequently, we believe that our current results will also be of high interest for the electrochemistry and battery application communities.

Magnetic Structure. The present system is composed of two types of magnetic ions, that is, Mn (HS: $\mathrm{d}^{4}$ for $\mathrm{Mn}^{3+}$ and HS: $\mathrm{d}^{5}$ for $\left.\mathrm{Mn}^{2+}\right)$ and $\mathrm{Fe}\left(\mathrm{HS}: \mathrm{d}^{5}\right.$ for $\mathrm{Fe}^{3+}$ ). The theoretical ordered magnetic moment values are $5.9 \mu_{\mathrm{B}}$ for $\mathrm{Fe}^{3+}$ and $4.9 \mu_{\mathrm{B}}$ for $\mathrm{Mn}^{3+}$. Consequently, the calculated values for each sublattice moments are $A=3.86$ and $B=3.70 \mu_{\mathrm{B}}$, as obtained by applying the cation distributions from the Rietveld refinement of the presented diffraction data (see Table 2). Clearly, the experimental (NPD) values for both $A$ and $B$ site magnetic moments $\left(A=1.57(5)\right.$ and $\left.B=1.22(4) \mu_{\mathrm{B}}\right)$ are well below theoretical expectations, losing $59.3 \%$ and $67.0 \%$ of the expected moment, respectively.

The exchange interaction within the cubic spinel family has already been extensively studied for related compounds by using both experimental and theoretical methods. ${ }^{26,49}$ Because of the unique geometry of the spinel structure, three types of magnetic interactions are expected. The strongest one is tetrahedra (A)-octahedra (B) antiferromagnetic interactions connected by the corner sharing $(\mathrm{A}-\mathrm{B}$ distance at $\mathrm{RT}=3.49$ $\AA$ ). The other two paths are expected to show relatively weak interplay: the edge-sharing $\mathrm{B}-\mathrm{B}(2.97 \AA)$ and the isolated AA $(3.64 \AA)$ interactions. Even if all three interactions are expected to be antiferromagnetic, the edge-sharing B-B spins favors a parallel ground state. In our study, the refined magnetic structure of $\mathrm{MgFeMnO}_{4}$ is in very good agreement with these expected exchange interactions. In $\mathrm{MgFeMnO}_{4}$, the $\mathrm{A}$ and $\mathrm{B}$ sites are occupied by two different magnetic atoms, which have different size of the magnetic moment, namely, $\mathrm{Mn}^{3+}$ and $\mathrm{Fe}^{3+}$. This yields two types of $\mathrm{A}-\mathrm{B}$ interactions: $\mathrm{Fe}-$ $\mathrm{Fe}$ or $\mathrm{Mn}-\mathrm{Mn}$ antiferromagnetic and $\mathrm{Fe}-\mathrm{Mn}$ ferrimagnetic. Because nonmagnetic $\mathrm{Mg}$ atoms are also distributed in both octahedral and tetrahedral sites, the magnetic ordering can be disturbed and instead form finite sized PM clusters. Because we observe clear magnetic Bragg peaks by NPD, we confirmed the existence of long-range magnetic ordering in this compound. However, the reduced magnetic moment on both $A$ and $B$ sites can be related to such PM clusters, which naturally do not contribute to the magnetic Bragg peaks 
(i.e., such a magnetic contribution is hidden in the background signal). This could potentially also explain the broadening observed in the Mössbauer spectra of Figure 2. On the other hand, if we have large PM clusters in our $\mathrm{MgFeMnO}_{4}$ sample, the wTF $\mu^{+}$SR spectra would show an oscillating volume fraction even below the magnetic transition temperature (which they clearly do not display). One possible explanation could be that the ferrimagnetic order induces a large enough resulting local field in such small PM clusters that the muon spin is still depolarized beyond the time resolution of the experimental setup. To potentially confirm such a scenario, we would need to conduct additional ZF $\mu^{+}$SR experiments using a high-time-resolution $\mu^{+}$SR setup (e.g., HAL-9500 instrument at PSI or NuTime spectrometer at TRIUMF).

Finally, another interesting point is that the concentration of diamagnetic ions in the $B$ site is larger than that in the $A$ site. As a result, the system becomes magnetically inhomogeneous and frustrated, causing a perturbation to the usual collinear ferrimagnetic ordering of spins. Previous studies of related compounds have revealed that the nonmagnetic cation concentration in the $A$ sites is critical for the formation of long-range magnetic ordering. For instance, the investigation of the cubic spinel $\mathrm{Zn}_{x} \mathrm{Mg}_{0.75-x} \mathrm{Cu}_{0.25} \mathrm{Fe}_{2} \mathrm{O}_{4}(x=0.75,70 \%$ of $A$ sites are occupied by nonmagnetic cation) only shows a shortrange magnetic ordering as confirmed by neutron powder diffraction. $^{50}$ The $\mathrm{Mg}$ concentration on $A$ in the case of $\mathrm{MgFeMnO}_{4}(33 \%)$ is clearly not high enough to destroy the long-range ordering. It should also be mentioned at this point that the geometrical frustration of the $B$ site due to the pyrochlore sublattice formed by the tetrahedral $B$ site may have some additional contribution to the strong disorder in the $B$ site, which is often observed in the spinel compound. ${ }^{43,51,52}$

\section{CONCLUSION}

Accurate studies of crystal structure of the magnesium spinel $\mathrm{MgFeMnO}_{4}$ have been performed by combining electron, Xray, and neutron diffraction techniques. The crystal structure has been confirmed to be a typical cubic spinel structure (SG: $F d \overline{3} m$ with $a=8.429 \AA$ at $500 \mathrm{~K})$. Three cations $(\mathrm{Mg}, \mathrm{Fe}$, and $\mathrm{Mn})$ are found to be distributed over both tetragonal $A$ and octahedral $B$ sites, and fractional occupancies are presented. These results confirm the presence of antisite defects and their importance for improved electrochemical performance in $\mathrm{MgFeMnO}_{4}$ and related compounds. In addition, the crystal structure is unaffected by the onset of the magnetic order, which is determined to be a collinear ferrimagnetic order by neutron powder diffraction. From $\mu^{+} \mathrm{SR}$ measurements it is found that the entire sample enters the ferrimagnetic state below the magnetic transition temperature $T_{\mathrm{C}}=394.2(12) \mathrm{K}$. In the ferrimagnetic phase, no muon spin oscillations are observed in the ZF- $\mu^{+}$SR time spectra, which is consistent with the ferrimagnetic spin ordering determined by neutron diffraction. Hence, the magnetic properties of $\mathrm{MgFeMnO}_{4}$ are more similar to those of the ferrimagnetic $\mathrm{MgFe}_{2} \mathrm{O}_{4}$ than to those of the antiferromagnetic $\mathrm{MgMn}_{2} \mathrm{O}_{4}$. Our findings provide new and improved understanding for the fundamental properties of $\mathrm{MgFeMnO}_{4}$. Consequently, the current research should also be of high importance for future potential applications within spintronics as well as battery devices.

\section{ASSOCIATED CONTENT}

\section{Supporting Information}

The Supporting Information is available free of charge at https://pubs.acs.org/doi/10.1021/acs.inorgchem.0c02241.

Detailed cation weight ratios obtained from ICP-AES method, images of $\mathrm{MgFeMnO}_{4}$ particles obtained by SEM and TEM, and the electron diffraction along the main zone axis [111] and simulated pattern indexed by the cubic structure; fitting result of Mössbauer measurement using both Lorentzian peak and pseudo-Voigt peak functions (ZIP)

\section{Accession Codes}

CCDC 2019621 and 2021028 contain the supplementary crystallographic data for this paper. These data can be obtained free of charge via www.ccdc.cam.ac.uk/data_request/cif, or by emailing data_request@ccdc.cam.ac.uk, or by contacting The Cambridge Crystallographic Data Centre, 12 Union Road, Cambridge CB2 1EZ, UK; fax: +44 1223336033.

\section{AUTHOR INFORMATION}

\section{Corresponding Authors}

Nami Matsubara - Department of Applied Physics, KTH Royal Institute of Technology, SE-10691 Stockholm, Sweden; ○ orcid.org/0000-0002-8324-710X; Email: namim@kth.se

Martin Månsson - Department of Applied Physics, KTH Royal Institute of Technology, SE-10691 Stockholm, Sweden; Email: condmat@kth.se

\section{Authors}

Titus Masese - Department of Energy and Environment, Research Institute of Electrochemical Energy (RIECEN) and AIST-Kyoto University Chemical Energy Materials Open Innovation Laboratory (ChEM-OIL), National Institute of Advanced Industrial Science and Technology (AIST), Ikeda, Osaka 563-8577, Japan; 이이.org/0000-0001-86949839

Emmanuelle Suard - Institut Laue-Langevin, 38042 Cedex 9 Grenoble, France; 이이.org/0000-0001-5966-5929

Ola Kenji Forslund - Department of Applied Physics, KTH Royal Institute of Technology, SE-10691 Stockholm, Sweden

Elisabetta Nocerino - Department of Applied Physics, KTH Royal Institute of Technology, SE-10691 Stockholm, Sweden

Rasmus Palm - Department of Applied Physics, KTH Royal Institute of Technology, SE-10691 Stockholm, Sweden

Zurab Guguchia - Laboratory for Muon Spin Spectroscopy, Paul Scherrer Institute, CH-5232 Villigen, PSI, Switzerland

Daniel Andreica - Faculty of Physics, Babes-Bolyai University, 400084 Cluj-Napoca, Romania

Alexandra Hardut - Faculty of Physics, Babes-Bolyai University, 400084 Cluj-Napoca, Romania

Motoyuki Ishikado - Comprehensive Research Organization for Science and Society (CROSS), Tokai, Ibaraki 319-1106, Japan

Konstantinos Papadopoulos - Department of Physics, Chalmers University of Technology, SE-41296 Gothenburg, Sweden

Yasmine Sassa - Department of Physics, Chalmers University of Technology, SE-41296 Gothenburg, Sweden

Complete contact information is available at: https://pubs.acs.org/10.1021/acs.inorgchem.0c02241 


\section{Notes}

The authors declare no competing financial interest.

\section{ACKNOWLEDGMENTS}

The authors thank S. Lipcsei (Babeş-Bolyai University, ClujNapoca, Romania) for his great support during the $\mu^{+} S R$ experiment. Magnetization measurement was performed at CROSS laboratory (Japan). The authors are grateful to ILL and Paul Scherrer Institute for the allocated neutron/muon beamtime as well as the great support from their technical staff. This research was supported by the Swedish Research Council (VR) through a Neutron Project Grant (Dnr. 2016-06955) as well as the Carl Tryggers Foundation for Scientific Research (CTS-18:272). T.M. is grateful to Prof. Daisuke Mori and Dr. Mitsuharu Tabuchi respectively for help in undertaking magnetic susceptibility and Mössbauer measurements. T.M. acknowledges the Nippon Sheet Glass Foundation for Materials Science and Engineering. Y.S. is funded by the Swedish Research Council (VR) through a Starting Grant (Dnr. 2017-05078) and K.P. and Y.S. acknowledge Chalmers Area of Advance - Materials Science. E.N. is fully funded by the Swedish Foundation for Strategic Research (SSF) within the Swedish national graduate school in neutron scattering (SwedNess). D.A. acknowledges partial financial support from the Romanian UEFISCDI Project No. PN-III-P4-IDPCCF-2016-0112.

\section{REFERENCES}

(1) Verma, S.; Joy, P. A.; Khollam, Y. B.; Potdar, H. S.; Deshpande, S. B. Synthesis of nanosized $\mathrm{MgFe} 2 \mathrm{O} 4$ powders by microwave hydrothermal method. Mater. Lett. 2004, 58, 1092-1095.

(2) Chen, Q.; Zhang, Z. J. Size-dependent superparamagnetic properties of $\mathrm{MgFe} 2 \mathrm{O} 4$ spinel ferrite nanocrystallites. Appl. Phys. Lett. 1998, 73, 3156-3158.

(3) De Renzi, R.; Allodi, G.; Mazzoli, C.; Ghigna, P.; Malavasi, L. Magnetic order and spin dynamics in substituted spinel $\mathrm{Mg} 1-$ xMn2+xO4. Phys. B 2003, 326, 509-512.

(4) Kondrat'eva, O. N.; Nikiforova, G. E.; Shevchenko, E. V.; Smirnova, M. N. Low-temperature magnetic properties of MgFe1.2Ga0.8O4 spinel nanoparticles. Ceram. Int. 2020, 46, 11390-11396.

(5) Orikasa, Y.; Masese, T.; Koyama, Y.; Mori, T.; Hattori, M.; Yamamoto, K.; Okado, T.; Huang, Z. D.; Minato, T.; Tassel, C.; Kim, J.; Kobayashi, Y.; Abe, T.; Kageyama, H.; Uchimoto, Y. High energy density rechargeable magnesium battery using earth-abundant and non-toxic elements. Sci. Rep. 2015, 4, 5622.

(6) Han, J.; Yagi, S.; Ichitsubo, T. Suppressive effect of Fe cations in $\mathrm{Mg}(\mathrm{Mn} 1 \mathrm{xFex}) 2 \mathrm{O} 4$ positive electrodes on oxidative electrolyte decomposition for $\mathrm{Mg}$ rechargeable batteries. J. Power Sources 2019, 435, 226822.

(7) Shimokawa, K.; Ichitsubo, T. Spinel-rocksalt transition as a key cathode reaction toward high-energy-density magnesium rechargeable batteries. Curr. Opin. Electrochem. 2020, 21, 93-99.

(8) Naaz, F.; Dubey, H. K.; Kumari, C.; Lahiri, P. Structural and magnetic properties of $\mathrm{MgFe} 2 \mathrm{O} 4$ nanopowder synthesized via coprecipitation route. SN Appl. Sci. 2020, 2, 808.

(9) Kumar, D.; Banerjee, A.; Mahmoud, A.; Rath, C. Cation distribution dependent magnetic properties in $\mathrm{CoCr} 2-\mathrm{XFexO} 4(\mathrm{x}=$ 0.1 to 0.5 ): EXAFS, Mössbauer and magnetic measurements. Dalton Trans 2017, 46, 10300-10314.

(10) Zeng, X.; Zhang, J.; Zhu, S.; Deng, X.; Ma, H.; Zhang, J.; Zhang, Q.; Li, P.; Xue, D.; Mellors, N. J.; Zhang, X.; Peng, Y. Direct observation of cation distributions of ideal inverse spinel $\mathrm{CoFe} 2 \mathrm{O} 4$ nanofibres and correlated magnetic properties. Nanoscale 2017, 9, $7493-7500$
(11) Kriessman, C. J.; Harrison, S. E. Cation distributions in ferrospinels. Magnesium-manganese ferrites. Phys. Rev. 1956, 103, $857-860$.

(12) Prasad, S.; Deepty, M.; Ramesh, P. N.; Prasad, G.; Srinivasarao, K.; Srinivas, C.; Vijaya Babu, K.; Ranjith Kumar, E.; Krisha Mohan, N.; Sastry, D. L. Synthesis of MFe2O4 (M = Mg2+, Zn2+, Mn2+) spinel ferrites and their structural, elastic and electron magnetic resonance properties. Ceram. Int. 2018, 44, 10517-10524.

(13) Manikandan, M.; Manimuthu, P.; Venkateswaran, C. Structural and magnetic properties of $\mathrm{MgFe} 2 \mathrm{O} 4$ ceramic. AIP Conf. Proc. 2013, 1576, 194-196.

(14) De Grave, E.; Govaert, A.; Chambaere, D.; Robbrecht, G. A Mössbauer effect study of $\mathrm{MgFe}_{2} \mathrm{O}_{4}$. Physica $B+C$ 1979, 96, 103-110.

(15) Manaila, R. Cation migration in tetragonal spinels (MgMn2O4). J. Phys. Chem. Solids 1967, 28, 2335-2341.

(16) Zhao, H.; Liu, L.; Xiao, X.; Hu, Z.; Han, S.; Liu, Y.; Chen, D.; Liu, X. The effects of Co doping on the crystal structure and electrochemical performance of $\mathrm{Mg}(\mathrm{Mn} 2-\mathrm{xCox}) \mathrm{O} 4$ negative materials for lithium ion battery. Solid State Sci. 2015, 39, 23-28.

(17) Malavasi, L.; Galinetto, P.; Mozzati, M. C.; Azzoni, C. B.; Flor, G. Raman spectroscopy of $\mathrm{AMn} 2 \mathrm{O} 4(\mathrm{~A}=\mathrm{Mn}, \mathrm{Mg}$ and $\mathrm{Zn})$ spinels. Phys. Chem. Chem. Phys. 2002, 4, 3876-3880.

(18) Rodríguez-Carvajal, J. Recent advances in magnetic structure determination by neutron powder diffraction. Phys. B 1993, 192, 5569.

(19) Aroyo, M. I.; Perez-Mato, J. M.; Capillas, C.; Kroumova, E.; Ivantchev, S.; Madariaga, G.; Kirov, A.; Wondratschek, H. Bilbao crystallographic server: I. Databases and crystallographic computing programs. Z. Kristallogr. - Cryst. Mater. 2006, 221, 15-27.

(20) Klencsár, Z.; Kuzmann, E.; Vértes, A. User-friendly software for Mössbauer spectrum analysis. J. Radioanal. Nucl. Chem. 1996, 210, $105-118$.

(21) MossWinn; http://www.mosswinn.com/english/index.html (Accessed 2019-08-21).

(22) Amato, A.; Luetkens, H.; Sedlak, K.; Stoykov, A.; Scheuermann, R.; Elender, M.; Raselli, A.; Graf, D. The new versatile general purpose surface-muon instrument (GPS) based on silicon photomultipliers for $\mu \mathrm{SR}$ measurements on a continuous-wave beam. Rev. Sci. Instrum. 2017, 88, 93301.

(23) Yaouanc, A.; Dalmas De Réotier, P. Muon Spin Rotation, Relaxation, and Resonance Applications to Condensed Matter; Oxford University Press: 2011.

(24) Suter, A.; Wojek, B. M. Musrfit: A Free Platform-Independent Framework for $\mu$ SR Data Analysis. Phys. Procedia 2012, 30, 69-73.

(25) O'Neill, H. S. C.; Annersten, H.; Virgo, D. The temperature dependence of the cation distribution in magnesioferrite (MgFe2O4) from powder XRD structural refinements and Mössbauer spectroscop. Am. Mineral. 1992, 77, 725-740.

(26) Zhang, X.; Kuang, X. J.; Wang, Y. G.; Wang, X. M.; Wang, C. H.; Zhang, Y.; Chen, C.; Jing, X. P. Transport and magnetic properties of MgFeVO4. Jpn. J. Appl. Phys. 2013, 52, 023001.

(27) Yusuf, S. M.; Rao, L. M. The magnetic domain effect in the local canted spin ferrite $\mathrm{Zn} 0.5 \mathrm{Co} 0.5 \mathrm{Fe} 2$-xCrxO4: A macroscopic and mesoscopic study. J. Phys.: Condens. Matter 1995, 7, 5891-5899.

(28) Yagi, S.; Ichikawa, Y.; Yamada, I.; Doi, T.; Ichitsubo, T.; Matsubara, E. Synthesis of Binary Magnesium - Transition Metal Oxides via Inverse Coprecipitation. Jpn. J. Appl. Phys. 2013, 52, 025501 .

(29) Bengtson, A.; Li, J.; Morgan, D. Mössbauer modeling to interpret the spin state of iron in $(\mathrm{Mg}, \mathrm{Fe}) \mathrm{SiO} 3$ perovskite. Geophys. Res. Lett. 2009, 36, L15301.

(30) Gütlich, P. Fifty years of Mössbauer spectroscopy in solid state research - Remarkable achievements, future perspectives. Z. Anorg. Allg. Chem. 2012, 638, 15-43.

(31) Dyar, M. D.; Sklute, E. C. In Remote Compositional Analysis: Techniques for Understanding Spectroscopy, Mineralogy, and Geochemistry of Planetary Surfaces; Bishop, J. L., Bell, III, J. F., Moersch, J. E., Eds.; Cambridge Planetary Science; Cambridge University Press: 2019; pp 147-167. 
(32) Lee, S. W.; Kim, S. J.; Kim, C. S. Superexchange interactions in $\mathrm{MgFe}_{2} \mathrm{O}_{4}$. J. Korean Phys. Soc. 2006, 48, 583-588.

(33) Osmond, W. Magnetic exchange mechanisms in magnesiummanganese ferrites. Proc. Inst. Electr. Eng., Part B 1957, 104, 221-227.

(34) Gateshki, M.; Petkov, V.; Pradhan, S. K.; Vogt, T. Structure of nanocrystalline $\mathrm{MgFe} 2 \mathrm{O} 4$ from X-ray diffraction, Rietveld and atomic pair distribution function analysis. J. Appl. Crystallogr. 2005, 38, 772779.

(35) Nakatsuka, A.; Ueno, H.; Nakayama, N.; Mizota, T.; Maekawa, H. Single-crystal X-ray diffraction study of cation distribution in MgAl2O4-MgFe2O4 spinel solid solution. Phys. Chem. Miner. 2004, 31, 278-287.

(36) Antao, S. M.; Hassan, I.; Parise, J. B. Cation ordering in magnesioferrite, $\mathrm{MgFe} 2 \mathrm{O} 4$, to $982^{\circ} \mathrm{C}$ using in situ synchrotron X-ray powder diffraction. Am. Mineral. 2005, 90, 219-228.

(37) Gauster, W. B.; Heffner, R. H.; Huang, C. Y.; Hutson, R. L.; Leon, M.; Parkin, D. M.; Schillaci, M. E.; Triftshäuser, W.; Wampler, W. R. Measurement of the depolarization rate of positive muons in copper and aluminum. Solid State Commun. 1977, 24, 619-622.

(38) Zhao, K.; et al. New diluted ferromagnetic semiconductor with curie temperature up to $180 \mathrm{~K}$ and isostructural to the '122' iron-based superconductors. Nat. Commun. 2013, 4, 1-5.

(39) Dunsiger, S. R.; Carlo, J. P.; Goko, T.; Nieuwenhuys, G.; Prokscha, T.; Suter, A.; Morenzoni, E.; Chiba, D.; Nishitani, Y.; Tanikawa, T.; Matsukura, F.; Ohno, H.; Ohe, J.; Maekawa, S.; Uemura, Y. J. Spatially homogeneous ferromagnetism of (Ga, Mn)As. Nat. Mater. 2010, 9, 299-303.

(40) Chang, H.; Hwang, I. Y.; Chung, J. H.; Stewart, J. R.; Higemoto, W.; Miyake, Y. Temperature dependence of magnetic excitations in the frustrated antiferromagnetic spinel $\mathrm{ZnMn2O} 4$. Phys. Rev. B: Condens. Matter Mater. Phys. 2018, 97, 014406 1-7.

(41) Balents, L. The impact of ionic frustration on electronic order. Science 2012, 336, 547-548.

(42) Tokura, Y.; Nagaosa, N. Orbital Physics in Transition-Metal Oxides. Science 2000, 288, 462-468.

(43) Radaelli, P. G. Orbital ordering in transition-metal spinels. New J. Phys. 2005, 7, 53.

(44) Shi, Y.; Ndione, P. F.; Lim, L. Y.; Sokaras, D.; Weng, T. C.; Nagaraja, A. R.; Karydas, A. G.; Perkins, J. D.; Mason, T. O.; Ginley, D. S.; Zunger, A.; Toney, M. F. Self-doping and electrical conductivity in spinel oxides: Experimental validation of doping rules. Chem. Mater. 2014, 26, 1867-1873.

(45) Sugiyama, J.; Ikedo, Y.; Mukai, K.; Nozaki, H.; Månsson, M.; Ofer, O.; Harada, M.; Kamazawa, K.; Miyake, Y.; Brewer, J. H.; Ansaldo, E. J.; Chow, K. H.; Watanabe, I.; Ohzuku, T. Lowtemperature magnetic properties and high-temperature diffusive behavior of $\mathrm{LiNiO} 2$ investigated by muon-spin spectroscopy. Phys. Rev. B: Condens. Matter Mater. Phys. 2010, 82, 224412.

(46) Månsson, M.; Sugiyama, J. Muon-spin relaxation study on Liand Na-diffusion in solids. Phys. Scr. 2013, 88, 068509.

(47) Park, K. Y.; Park, I.; Kim, H.; Lim, H. D.; Hong, J.; Kim, J.; Kang, K. Anti-site reordering in LiFePO4: Defect annihilation on charge carrier injection. Chem. Mater. 2014, 26, 5345-5351.

(48) Tokur, M.; Erdas, A.; Nalci, D.; Oguz Guler, M.; Akbulut, H. 33V0.33Mn0.33O2 semiconductor as a cathode electrode for high rate Li-ion batteries. Mater. Sci. Semicond. Process. 2015, 38, 387-391.

(49) Ugendar, K.; Samanta, S.; Rayaprol, S.; Siruguri, V.; Markandeyulu, G.; Nanda, B. R. Effect of frustrated exchange interactions and spin-half-impurity on the electronic structure of strongly correlated NiFe2 O4. Phys. Rev. B: Condens. Matter Mater. Phys. 2017, 96, 1-11.

(50) Yunus, S. M.; Shim, H. S.; Lee, C. H.; Asgar, M. A.; Ahmed, F. U.; Zakaria, A. K. Neutron diffraction studies of the diluted spinel ferrite $\mathrm{ZnxMg0.75-xCu0.25Fe2O4.} \mathrm{J.} \mathrm{Magn.} \mathrm{Magn.} \mathrm{Mater.} \mathrm{2001,} \mathrm{232,}$ $121-132$.

(51) Reig-I-Plessis, D.; Cote, A.; Van Geldern, S.; Mayrhofer, R. D.; Aczel, A. A.; Macdougall, G. J. Neutron scattering measurement of crystalline-electric fields in magnesium rare-earth selenide spinels. Phys. Rev. Mater. 2019, 3, 1-12.
(52) Tomiyasu, K.; Fukunaga, J.; Suzuki, H. Magnetic short-range order and reentrant-spin-glass-like behavior in $\mathrm{CoCr} 2 \mathrm{O} 4$ and $\mathrm{MnCr} 2 \mathrm{O} 4$ by means of neutron scattering and magnetization measurements. Phys. Rev. B: Condens. Matter Mater. Phys. 2004, 70, $1-12$. 